\title{
Tri11, tri3, and tri4 genes are required for trichodermin biosynthesis of Trichoderma brevicompactum
}

\author{
Xuping Shentu ${ }^{1 \dagger}$, Jiayi Yao ${ }^{1 \dagger}$, Xiaofeng Yuan ${ }^{1}$, Linmao He ${ }^{1}$, Fan Sun ${ }^{1}$, Kozo Ochi ${ }^{2}$ and Xiaoping Yu${ }^{1 *}$
}

\begin{abstract}
Trichoderma brevicompactum and T. arundinaceum both can synthesize trichodermin with strong antifungal activity and high biotechnological value. The two Trichoderma species have a tri cluster, which includes seven genes (tri14, tri12, tri11, tri10, tri3, tri4, and tri6) that encode transport and regulatory enzymes required for the biosynthesis of trichodermin. Here, we isolated T. brevicompactum 0248 transformants with disrupted tri11, tri4, or tri3 gene. We also described the effect of tri11, tri3, or tri4 deletion on the expression of other genes in the tri cluster. Targeted $\Delta$ tri3 knockout mutant exhibited a sharp decline in the production of trichodermin, and trichodermol, which is a substrate for trichodermin production, accumulated. Thus, the results demonstrated that tri3 was responsible for the biosynthesis of trichodermin, and the tri3 gene-encoded enzyme catalyzed the acetylation reaction of the hydroxy group at C-4 of the trichodermin skeleton. In addition, tri4 and tri11 deletion mutants were generated to evaluate the roles of tri4 and tri1 1 in trichodermin biosynthesis, respectively. Deletion mutant strain $\Delta$ tri4 or $\Delta$ tri1 1 did not produce trichodermin in T. brevicompactum, indicating that tri4 and tri11 are essential for trichodermin biosynthesis. This is the first to report the function of tri3, tri4 and tri11 in T. brevicompactum, although the role of tri4 and tri1 1 has already been described for T. arundinaceum by Cardoza et al. (Appl Environ Microbiol 77:4867-4877, 2011).
\end{abstract}

Keywords: Trichoderma brevicompactum, tri3, tri4, tri11, Gene deletion, Trichodermin

\section{Introduction}

Trichoderma species are well-known biological control agents of diseases in numerous crops, and these species produce many antifungal compounds and cell-walldegrading enzymes (Harman 2006; Malmierca et al. 2012; Tijerino et al. 2011a, b). The biocontrol activities of Trichoderma spp. against phytopathogenic fungi generally include antibiosis, parasitism, and competition for space and nutrients (Harman 2006). Antibiotic molecules synthesized by Trichoderma are low-molecular-weight and volatile metabolites as well as high-molecular weight polar metabolites. The former type includes simple aromatic compounds, polyketides, volatile terpenes, and

\footnotetext{
*Correspondence: yxp@cjlu.edu.cn

${ }^{+}$Xuping Shentu and Jiayi Yao contributed equally to this work

1 Zhejiang Provincial Key Laboratory of Biometrology and Inspection

\& Quarantine, College of Life Sciences, China Jiliang University,

Hangzhou 310018, Zhejiang, China

Full list of author information is available at the end of the article
}

isocyanide metabolites, while the latter includes peptaibols and diketopiperazine-like gliotoxin and gliovirin compounds (Reino et al. 2008; Szekeres et al. 2005; Tijerino et al. 2011a). Trichothecenes belong to a large group of terpenoid-derived secondary metabolites and are mainly synthesized by Fusarium and other fungal genera, such as Trichoderma, Myrothecium, Spicellum, Stachybotrys, and Trichothecium (Shentu et al. 2014a; Wilkins et al. 2003). Terpenes are derived from the repetitive fusion of branched five-carbon units based on an isopentane skeleton, and most of the chemical intermediates in their biosynthetic pathway have been identified (Tijerino et al. 2011b). The trichothecene biosynthetic pathway in Fusarium has been documented and extensively reviewed (Kimura et al. 2007). However, the genes involved in trichothecene biosynthesis in the other genera remain unknown.

Trichodermin and harzianum A (HA), which are synthesized by $T$. brevicompactum and T. arundinaceum, 
belong to terpenes and have similar structures except for the side chain group at C-4 (an acetyl group and an octa-2,4,6-trienedioic acid, respectively) (Malmierca et al. 2012). T. arundinaceum has been used as a model to study the beneficial effect of trichothecenes on the Trichoderma biocontrol activity and modulation of plant defense responses of this fungus (Malmierca et al. 2012). Furthermore, bioactivity assays have shown that trichodermin synthesized by $T$. brevicompactum exhibits stronger antifungal activity against Saccharomyces cerevisiae, Kluyveromyces marxianus, Candida albicans, Aspergillus fumigatus, Botrytis cinereal, and Rhizoctonia solani than amphotericin B and hygromycin (Shentu et al. 2014a; Tijerino et al. 2011a). The mechanism and genes involved in the trichothecene biosynthesis in Trichoderma have been increasingly investigated. Although Fusarium and Trichoderma can synthesize trichothecenes, these species have remarkably different organizations of genes in the tri cluster and trichothecenes biosynthesis (Cardoza et al. 2011). TRI gene orthologues (tri) in T. arundinaceum and T. brevicompactum had been identified and characterized. The result showed that both Trichoderma species have a tri cluster with seven homologous genes in the Fusarium TRI cluster (Cardoza et al. 2011). Furthermore, the two Trichoderma species have the same organizations of genes in the tri cluster but different from that in Fusarium (Cardoza et al. 2011). Sequence and functional analyses demonstrated that the gene (tri5) responsible for the first step in trichothecene biosynthesis is located outside the cluster in both Trichoderma species but inside the cluster in Fusarium (Cardoza et al. 2011). Thus, analysis of the heterologous gene expression indicates that the two T. arundinaceum cluster genes (tri4 and tri11) differ in function from their Fusarium orthologues (Cardoza et al. 2011). The Tatri4-encoded enzyme catalyzes only three of the four oxygenation reactions catalyzed by the orthologous enzyme in Fusarium (Cardoza et al. 2011). By contrast, the Tatri4-encoded enzyme in $T$. arundinaceum has the same function as that of MrTri4-encoded enzyme in Myrothecium (McCormick and Alexander 2007). The Tatri11-encoded enzyme catalyzes a reaction (trichothecene C-4 hydroxylation) that is completely different from that of the Fusarium orthologue (trichothecene C-15 hydroxylation) (Cardoza et al. 2011). However, the function of tri3 gene in T. arundinaceum remains ambiguous. The lateral moiety at the $\mathrm{C}-4$ position of the $\mathrm{HA}$ might be added to trichodermol through acetylation using an acyltransferase encoded by the tri3 gene (Cardoza et al. 2011). The tri5 gene has a significant role in the production of trichodermin in T. brevicompactum such that its overexpression increases trichodermin production and antimicrobial activity (Tijerino et al.2011a, b). However, whether tri3 participates in the trichodermin biosynthesis remains unknown. The functions of tri11 and tri4 in T. brevicompactum should be verified. These genes are orthologues with Tatri11 and Tatri4 of $T$. arundinaceum.

We have identified and characterized the tri cluster of $T$. brevicompactum 0248, which can biosynthesize trichodermin (Yuan et al. 2016). The results indicated that $T$. brevicompactum 0248 has a 24,793 bp cluster that includes tri14, tri12, tri11, tri10, tri3, tri4, and tri6 genes which are highly homologous to those in the T. arundinaceum tri cluster (Yuan et al. 2016). Furthermore, the tri cluster in strain 0248 is highly homologous to that of the reported strain T. brevicompactum IBT 40841. Strain 0248 and IBT 40841 clusters primarily differ in the size of the tri11-tri12 intergenic region, which is $2287 \mathrm{bp}$ in strain 0248 and 3000 bp in IBT 40841. In this study, we described the effects of the disruption of tri11, tri3, and tri4 in T. brevicompactum 0248 on trichodermin production and gene expression of the other tri genes in trichodermin biosynthesis.

\section{Materials and methods}

Strains, culture media, and culture conditions

Trichoderma brevicompactum 0248 was isolated in our previous study and deposited in the China General Microbiological Culture Collection Center (CGMCC 6985) (Shentu et al. 2014b). The isolate was maintained on a potato-dextrose agar (PDA) slant medium at $4{ }^{\circ} \mathrm{C}$ until used.

Agrobacterium tumefaciens AGL-1, which was obtained from the Zhejiang Provincial Key Laboratory of Biometrology and Inspection and Quarantine, was grown in YEB medium with $100 \mu \mathrm{g} / \mathrm{mL}$ rifampicin at $28{ }^{\circ} \mathrm{C}$ before use for $A$. tumefaciens-mediated transformation (ATMT) (Lacorte et al. 1991). The tri11, tri3, and tri4 genes in the tri cluster of $T$. brevicompactum 0248 were deleted by homologous recombination method as previously described (Brown et al. 2004; Kumar 2010).

The plasmid pSilent-1, which was provided by the Fungal Genetics Stock Center in USA, carried a hygromycin-resistant gene $(h p h)$ cassette. The plasmid pCAMBIA0380, which was obtained from the Zhejiang Provincial Key Laboratory of Biometrology and Inspection and Quarantine, was used for ATMT.

All primers were synthesized by Shanghai Sunny Biotechnology Co. Ltd and are listed in Additional file 1: Table S1, Additional file 2: Table S2, Additional file 3: Table S3.

\section{Construction of recombinant vector}

First, using plasmid pSilent- 1 as template, $h p h$ was amplified by PCR with primers Ph-F/Ph-R (Additional file 1: 
Table S1) to obtain the $h p h$ expression cassette. Then, $1-\mathrm{kb}$ upstream DNA fragment before the tri11, tri3, and tir4 gene start codon was amplified using total DNA as template and corresponding primers P11-5F/P11-5R, P3-5F/P3-5R, and P4-5F/P4-5R. The forward primers P11-5F, P3-5F, and P4-5F contained the BstXI site, while the reverse primers P11-5R, P3-5R, and P4-5R contained a 22 bp reverse complementary sequence of the $h p h$ cassette. Similarly, another DNA fragment downstream of the tri11, tri3, and tir4 gene stop codon was amplified using primers $\mathrm{P} 11-3 \mathrm{~F} / \mathrm{P} 11-3 \mathrm{R}, \mathrm{P} 3-3 \mathrm{~F} / \mathrm{P} 3-3 \mathrm{R}$, and $\mathrm{P} 4-3 \mathrm{~F} /$ P4-3R, respectively. The forward primers P11-3F, P3-3F, and P4-3F contained a 22 bp overlapping sequence of the $h p h$ gene, while the reverse primers P11-3R, P3-3R, and P4-3R contained an XmaI site (Fig. 1). Then, the above three linearized fragments were equimolarly mixed and cycled in a fusion PCR to generate a gene knockout fragment (Cao et al. 2014). The corresponding gene knockout fragments were cloned into the BstXI/XmaI sites of pCAMBIA0380 to generate the corresponding expression constructs designated as pKT11, pKT3, and pKT4 (Fig. 1).

\section{Transformation of T. brevicompactum 0248}

The expression vectors pKT11, pKT3, and pKT4 were transformed using ATMT as described previously (Dos et al. 2004; Yang et al. 2011). The obtained transformants were confirmed by PCR and subcultured in PDA plates with $100 \mu \mathrm{g} / \mathrm{mL}$ hygromycin $\mathrm{B}$ for three generations to test the genetic stability.

\section{Quantitative RT-PCR (QRT-PCR) analysis}

Total RNA of T. brevicompactum 0248 and $\Delta$ tri11, $\Delta$ tri3, and $\Delta$ tri4 knockout strains were isolated using Spin Column Fungal Total RNA Purification Kit (Sangon, Shanghai, China). The total cDNA was obtained through reverse-transcription reaction using PrimeScript ${ }^{\circledR}$ RT Reagent Kit with gDNA Eraser (TaKaRa, Dalian, China). QRT-PCR was performed in an Applied Biosystems StepOnePlus Real-Time PCR System using a SYBR ${ }^{\circledR}$ Premix Ex TaqTM (TliRHaseH Plus) reagent (TaKaRa, Dalian, China) (Shentu et al. 2014a). The primers used in qRTPCR are listed in Additional file 2: Table S2. The acceptable qRT-PCR standard curve $\left(0.95 \leq \mathrm{E} \leq 1.05, \mathrm{R}^{2} \geq 0.99\right)$ of the gene examined in this study was optimized by

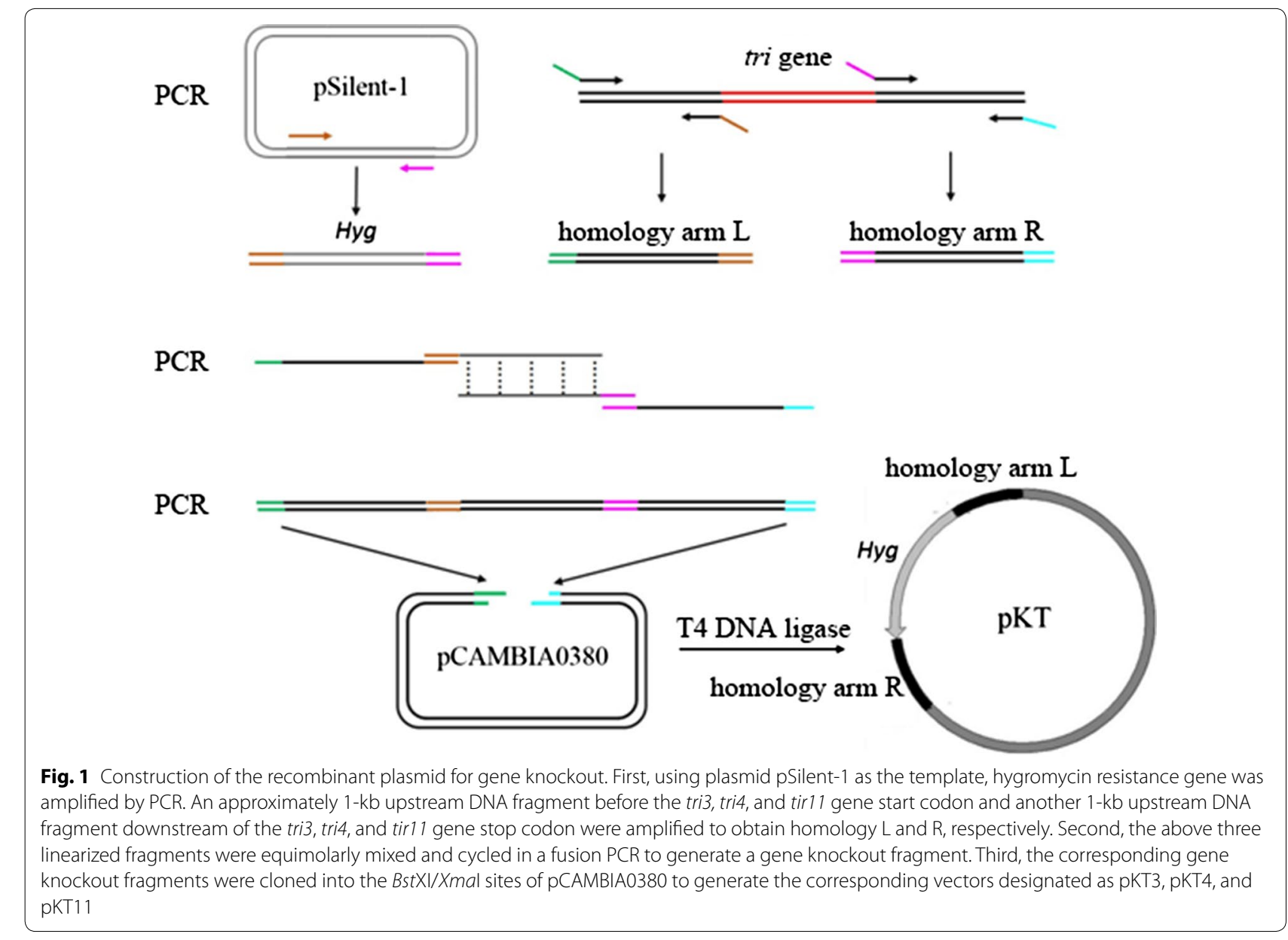


varying the annealing temperature and annealing time. The $\beta$-tubulin gene of T. brevicompactum 0248 was used as the reference gene (Shentu et al. 2014a). Quantification of the relative gene expression was analyzed by the $2^{-\Delta \Delta \mathrm{Ct}}$ method.

\section{Analysis of trichodermin using gas chromatography (GC)}

The fermentation broth was separated from the mycelia by filtration using a Buchner funnel and extracted exhaustively with ethyl acetate (v/v, 1:2). The organic fractions were combined and evaporated to dryness in a vacuum at $50{ }^{\circ} \mathrm{C}$. The recovered residues were resuspended in methanol and analyzed using GC to quantify trichodermin (Shentu et al. 2008).

\section{Results}

Isolation of tri11, tri3, and tri4 deletion mutants

Using the gene-deletion plasmid and ATMT, transformants were selected on PDA plates with $100 \mu \mathrm{g} / \mathrm{mL}$ hygromycin B. Then, these transformants were further identified by PCR using the primers $\mathrm{P} 11 \mathrm{~N}-\mathrm{R} / \mathrm{P} 11 \mathrm{~N}$ F, P3N-R/P3N-F, and P4N-R/P4N-F (Additional file 3: Table S3) to verify $\Delta$ tri11, $\Delta$ tri3, and $\Delta$ tri4 mutants, respectively. Figure $2 \mathrm{a}$ shows that 10 transformants were arbitrarily chosen for PCR analysis, of which transformants 2, 5, 6, and 10 (lanes 2, 5, 6, and 10, respectively) did not exhibit the expected $1.1 \mathrm{~kb}$ band. Furthermore, primer pair P3W-R/P3W-F was designed according to the flanking sequence of the upstream/downstream DNA fragments of tri3. This pair was used to verify whether transformants $2,5,6$, and 10 had the expected $3.6 \mathrm{~kb}$ band. The length of the hph cassette and ORF of the tri3 were 1.1 and $1.78 \mathrm{~kb}$, respectively. The band of lane 22 from the fragment of the wild strain was $4.3 \mathrm{~kb}$. If the ORF of the tri3 was replaced successfully by the $h p h$ cassette in mutants, transformants $2,5,6$, and 10 (lanes 13, 16, 17, and 21, respectively) should show the expected $3.6 \mathrm{~kb}$ band (reduced by approximately $0.7 \mathrm{~kb}$ ). These expected results were observed (Fig. 2a), indicating that $\Delta$ tri3 mutants were obtained successfully. $\Delta$ tri11 and $\Delta$ tri4 mutants were verified with the same method (Figs. 2b and c). Four transformants (lanes 1, 4, 5, and 7) in which the hph cassette replaced the ORF of the tri11 gene were identified (Fig. 2b). Five transformants (lanes $3,4,6,8$, and 9) in which the $h p h$ cassette replaced the ORF of the tri4 gene were also determined (Fig. 2c).

\section{Effect of tri3 deletion on the expression of the tri genes}

The relative expression levels of the tri genes in the $\Delta t r i 3$ mutant were analyzed and compared with those of the wild-strain 0248 . The expression of the tri3 gene in the wild strain changed with culture time $(16,28,40,52,64$, 76 , and $88 \mathrm{~h}$ ), with the highest expression level observed at fermentation for $40 \mathrm{~h}$ (Fig. 3a). The tri3 expression was not detected in the $\Delta$ tri3 mutant at each time point. Surprisingly, the deletion of tri3 resulted in the significant upregulation of the expression of tri4, tri5, tri6, tri10, tri11, tri12, and tri14 genes compared with that of the wild type after $40 \mathrm{~h}$ of culture. The expression of these genes in $\Delta$ tri3 mutant had remarkably declined from 52 to $88 \mathrm{~h}$ (Fig. 3).

\section{Effect of tri4 deletion on the expression of the tri genes}

We determined whether the deletion of tri4 gene affected the transcription of tri genes. The relative expression levels of tri3, tri4, tri5, tri6, tri10, tri11, tri12, and tri14 in the $\Delta$ tri4 mutant and wild strain 0248 were detected. The tri4 expression was not detected in the $\Delta$ tri4 mutant at each time point (Fig. 4). The deletion of tri4 positively affected the expression of tri3, tri5, tri6, tri10, tri11, tri12, and tri14 compared with the wild-type strain at $40 \mathrm{~h}$. After $52 \mathrm{~h}$ of fermentation, the expression levels of these genes declined.

\section{Effect of tri11 deletion on expression of the tri genes}

The tri11 deletion process was successful, and no expression of tri11 was found in the $\Delta$ tri11 mutant. After $52 \mathrm{~h}$ of culture, the expression level of tri3 was higher in the $\Delta$ tri11 mutant than in the wild strain. At other time points, relative expression levels of tri3, tri4, tri5, tri6, tri10, tri12, and tri14 in the $\Delta$ tri11 mutant were higher than in the wild strain (Fig. 5).

\section{Effect of tri3, tri4, or tri11 deletion on trichodermin production}

Given that trichodermin is the sole product of the tri cluster in T. brevicompactum 0248, the concentrations of trichodermin in the culture extracts were analyzed by GC in both wild strain and $\Delta$ tri gene mutants. No trichodermin was detected in the $\Delta$ tri4 and $\Delta$ tri11 mutants. That is, tri4 or tri11 deletion directly affected trichodermin biosynthesis by $T$. brevicompactum. However, $\Delta$ tri3 mutant could still biosynthesize trichodermin, although tri3 gene was deleted (Fig. 6a). Prolonged cultivation time resulted in increased trichodermin concentration in the $\Delta$ tri3 mutant and strain 0248 , although the trichodermin yield by $\Delta$ tri3 mutant was lower than that of the wild strain at $76 \mathrm{~h}$ of fermentation by approximately $40 \mathrm{mg} / \mathrm{L}$ trichodermin. Deletion of tri3 gene resulted in reduced trichodermin content. Furthermore, high abundance of trichodermol in the $\Delta$ tri3 strain was detected after $40 \mathrm{~h}$ cultivation, contrary to the quite low trichodermol content in the wild-type culture extracts (Fig. 6b). It was shown that trichodermol was accumulated in the culture broth of $\Delta$ tri3 mutant. 

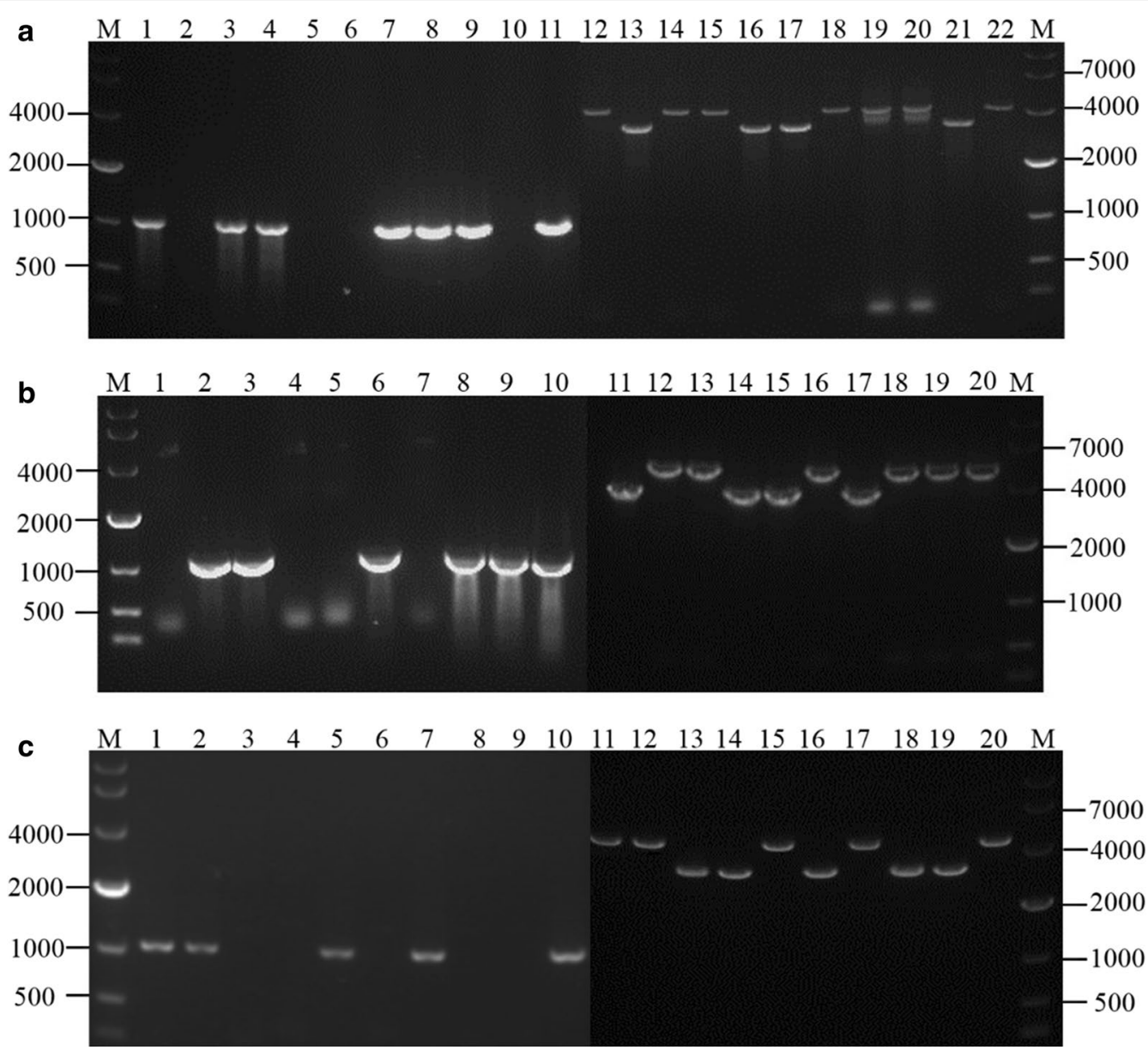

Fig. 2 Agar gel analysis. a Verification of tri3 deletion. Numbers from 1 to 10 and 12 to 21 refer to the arbitrarily chosen transformants. Numbers 11 and 22 refer to the wild strain. The numbers and their corresponding transformants are as follows: 1 and $12, \Delta$ tri3-1;2 and 13, $\Delta$ tri3-2;3 and 14, $\Delta$ tri33; 4 and 15, $\Delta$ tri3-4; 5 and 16, $\Delta$ tri3-5; 6 and 17, $\Delta$ tri3-6; 7 and 18, $\Delta$ tri3-7; 8 and 19, $\Delta$ tri3-8; 9 and 20, $\Delta$ tri3-9; and 10 and 21, $\Delta$ tri3-10. Lanes 2, 5, 6, and 10 did not show the expected band of $1.1 \mathrm{~kb}$. This result indicated that tri 3 was deleted in $\Delta$ tri3-2, $\Delta$ tri3-5, $\Delta$ tri3-6, and $\Delta$ tri3-10, respectively. Furthermore, $\Delta$ tri3-2, $\Delta$ tri3-5, $\Delta$ tri3-6, and $\Delta$ tri3-10 (lanes 13, 16, 17, and 21, respectively) showed the expected band of $3.6 \mathrm{~kb}$ (reduced by approximately $0.7 \mathrm{~kb}$ ). The ORF of the tri3 was replaced successfully by the hygromycin cassette in these mutants. $\mathbf{b}$ Verification of tril1 deletion. Numbers from 1 to 9 and 11 to 19 refer to the arbitrarily chosen transformants, while numbers 10 and 20 refer to the wild strain. The numbers and their correspond-

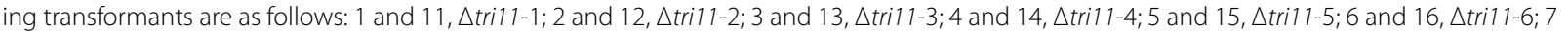
and 17, $\Delta$ tri 11-7; 8 and 18, $\Delta$ tri 11-8; and 9 and 19, $\Delta$ tri 1 1-9. Lanes 1, 4, 5, and 7 did not show the expected band of $1.1 \mathrm{~kb}$. This result indicated that tri1 1 was deleted in $\Delta$ tri 1 1-1, $\Delta$ tri 1 1-4, $\Delta$ tri1 1-5, and $\Delta$ tri 1 1-7, respectively. Furthermore, $\Delta$ tri1 1-1, $\Delta$ tri 1 1-4, $\Delta$ tri 1 1-5, and $\Delta$ tri1 1-7 (lanes $11,14,15$, and 17 , respectively) showed the expected band of $3.6 \mathrm{~kb}$ (reduced by approximately $0.7 \mathrm{~kb}$ ). The ORF of the tri11 was replaced successfully by the hygromycin cassette in these mutants. c Verification of tri4 deletion. Numbers from 1 to 9 and 11 to 19 refer to the arbitrarily chosen transformants, while numbers 10 and 20 refer to the wild strain. The numbers and their corresponding mutants are as follows: 1 and $11, \Delta$ tri4-1;2 and 12, $\Delta$ tri4-2; 3 and $13, \Delta$ tri4-3; 4 and $14, \Delta$ tri4-4; 5 and $15, \Delta$ tri4-5; 6 and $16, \Delta$ tri4-6; 7 and 17, $\Delta$ tri4-7; 8 and 18, $\Delta$ tri4-8; and 9 and 19, tri4-9. Lanes 3, 4, 6, 8, and 9 did not show the expected band of $1.1 \mathrm{~kb}$. This result indicated that tri4 was deleted in $\Delta$ tri4-3, $\Delta$ tri4-4, $\Delta$ tri4-6, $\Delta$ tri4-8, and $\Delta$ tri4-9, respectively. Furthermore, $\Delta$ tri4-3, $\Delta$ tri4-4, $\Delta$ tri4-6, $\Delta$ tri4-8, and $\Delta$ tri4-9 (lanes $13,14,16,18$, and 19, respectively) showed the expected band of $3.6 \mathrm{~kb}$ (reduced by approximately $0.7 \mathrm{~kb}$ ). The ORF of the tri4 was replaced successfully by the hygromycin cassette in these mutants

\section{Discussion}

Trichoderma is an interesting fungus because of its important application in biocontrol (Malmierca et al. 2012). However, T. brevicompactum has not been wellstudied among Trichoderma, because T. brevicompactum (IBT 9471) had been erroneously considered as $T$. harzianum (ATCC 90237) because of substantial shared micromorphology of these two species (Nielsen et al. 2005; Degenkolb et al. 2008). Until 2005, this IBT 9471 strain has been reclassified as T. brevicompactum on the basis of phylogenetic lineage within the morphological species T. brevicompactum and trichothecene production (Nielsen et al. 2005; Shentu et al. 2014a). Previous studies reported that $T$. brevicompactum is one of the 

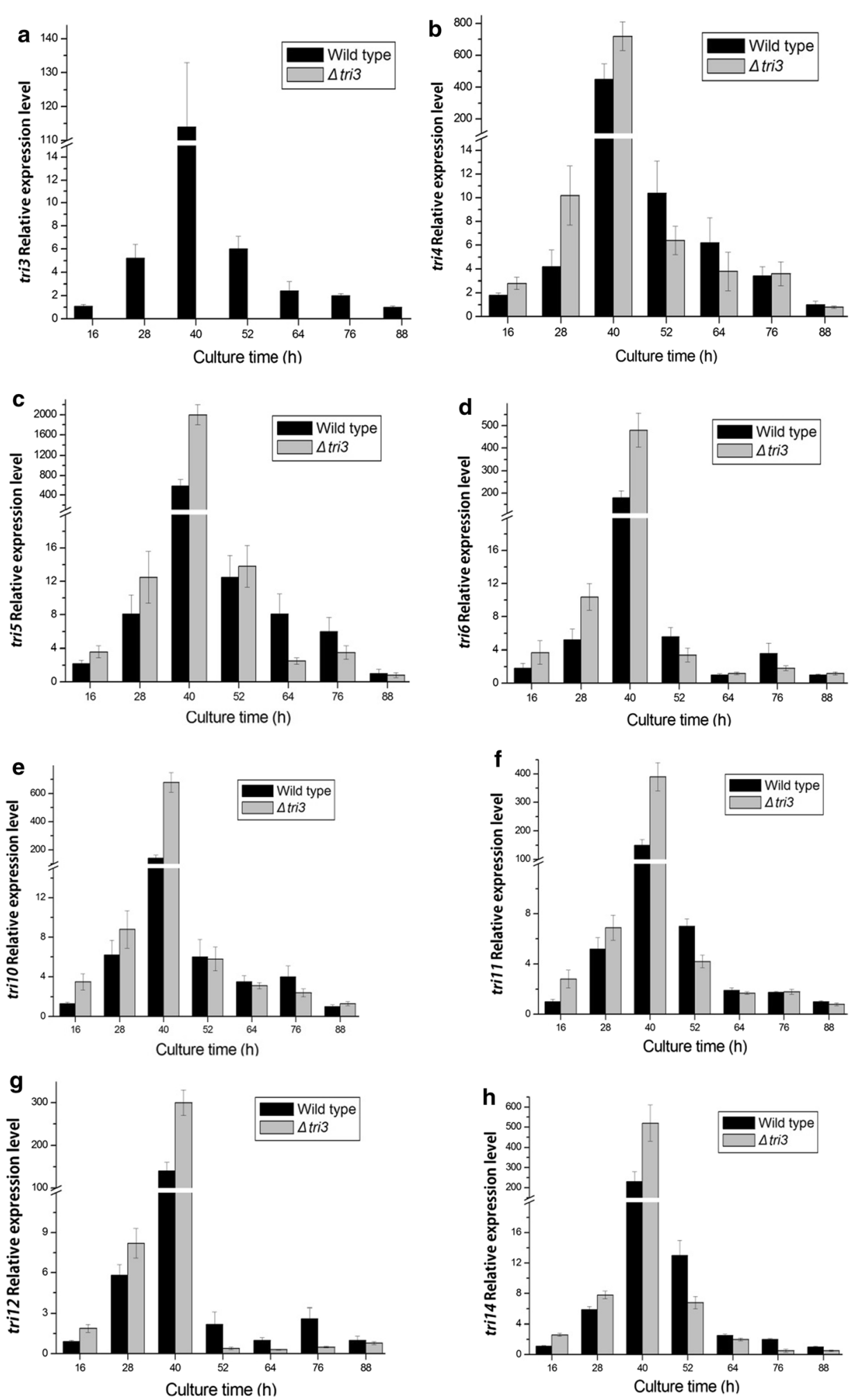

Fig. 3 Expression of tri genes in $\triangle$ tri3 mutant and wild strain. The quantification of tri3 gene expression in the different culture times was analyzed by $2^{-\Delta \Delta \mathrm{ct}}$ method. The wild strain cultured for $88 \mathrm{~h}$ was used as control and $\beta$-tubulin as reference gene. a tri3, $\mathbf{b}$ tri4, $\mathbf{c}$ tri5, $\mathbf{d}$ tri6, e tri10, f-tri1 1, $\mathbf{g}$ tri12, and $\mathbf{h}$ tri14 

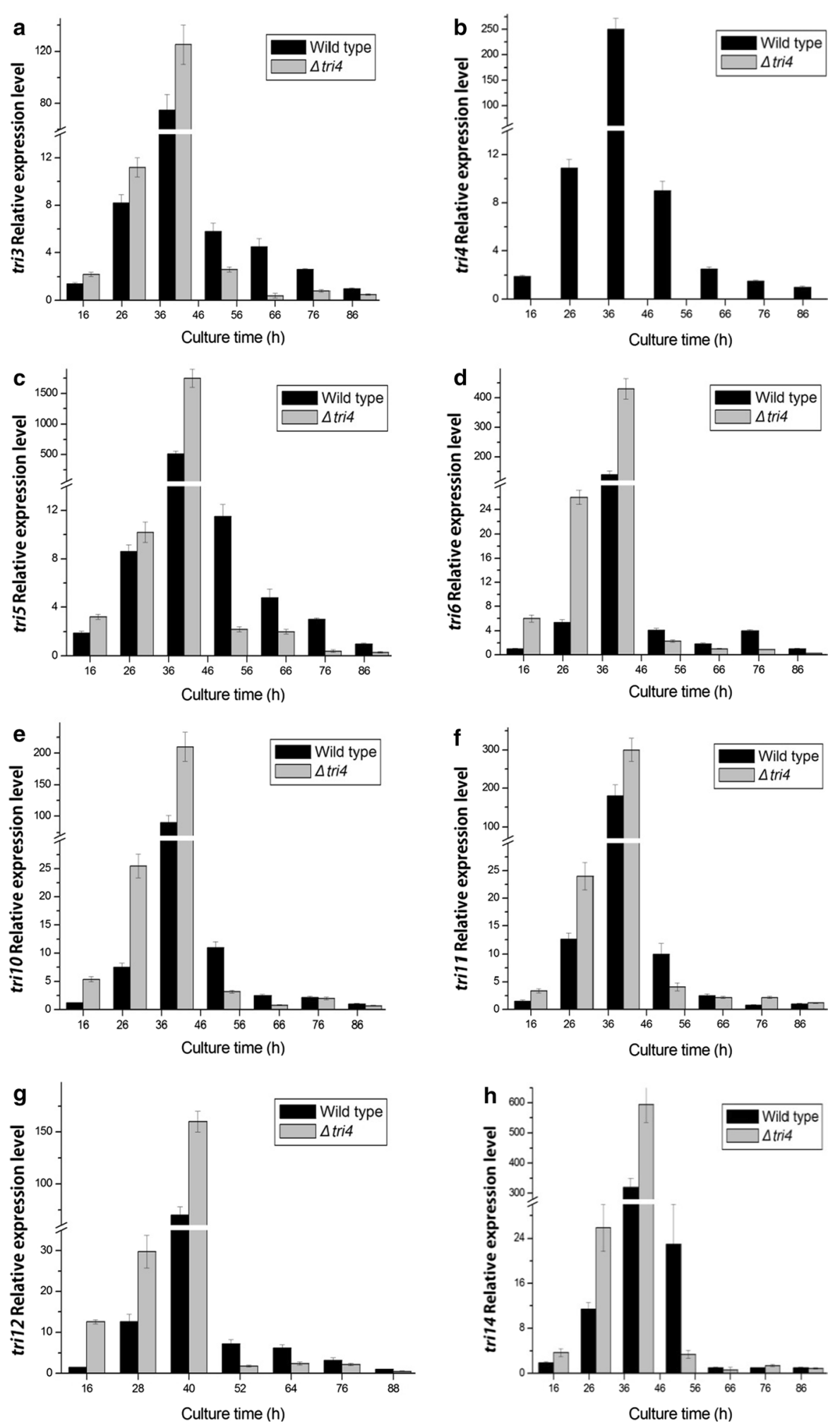

Fig. 4 Expression of tri genes in $\triangle$ tri4 mutant and wild strain. The quantification of tri4 gene expression in the different culture times was analyzed by $2^{-\Delta \Delta C t}$ method. The wild strain cultured for $88 \mathrm{~h}$ was used as control and $\beta$-tubulin as reference gene. a tri3, $\mathbf{b}$ tri4, $\mathbf{c}$ tri5, $\mathbf{d}$ tri6, e tri10, f tri11, $\mathbf{g}$ tri12, and $\mathbf{h}$ tri14 

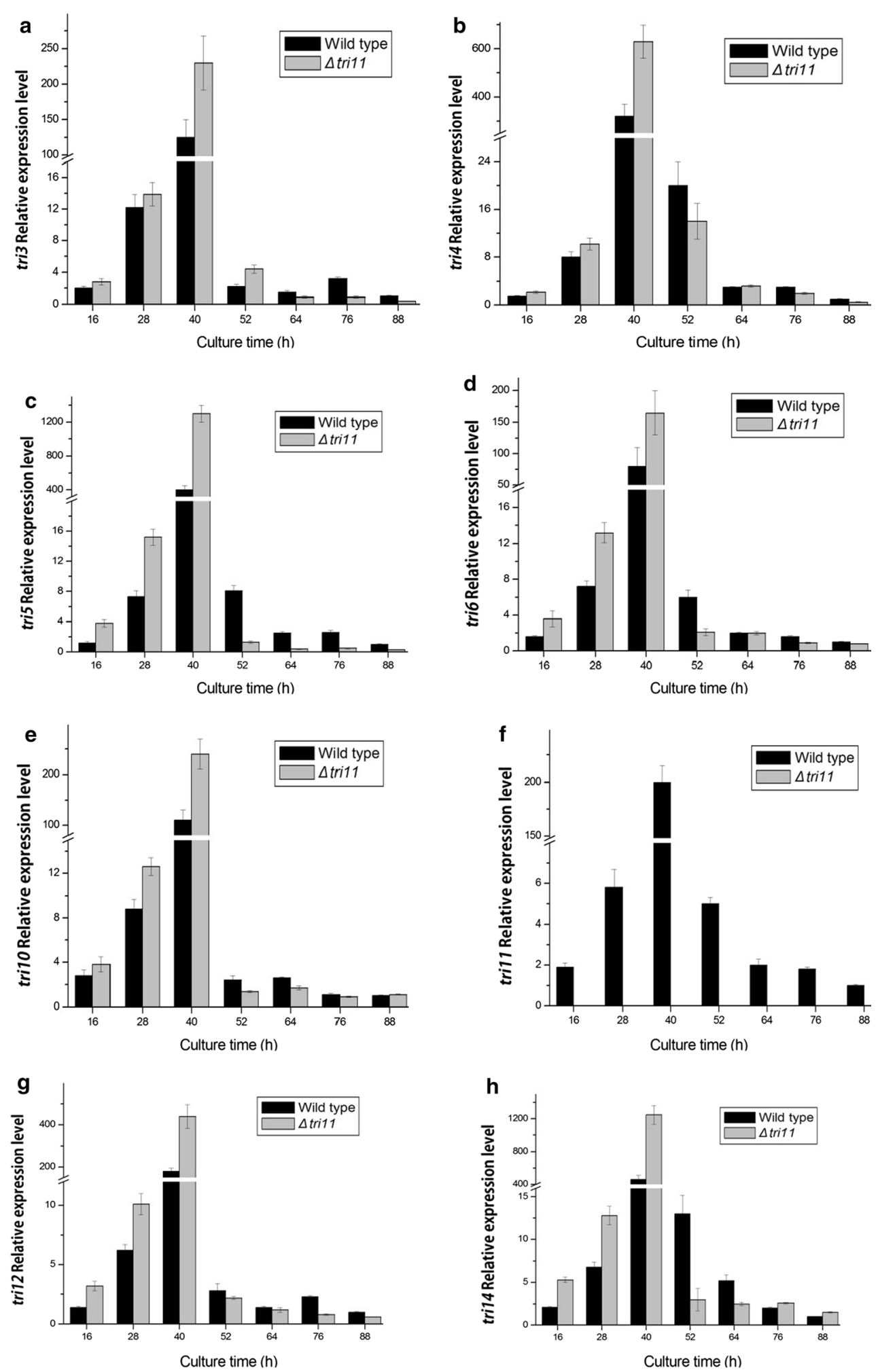

Fig. 5 Expression of tri genes in $\triangle$ tri 11 mutant and wild strain. The quantification of tri1 1 gene expression in the different culture times was analyzed by $2^{-\Delta \Delta \mathrm{Ct}}$ method. The wild strain cultured for $88 \mathrm{~h}$ was used as control and $\beta$-tubulin as reference gene. a tri3, b tri4, $\mathbf{c}$ tri5, $\mathbf{d}$ tri6, e tri10, $\mathbf{f}$ tri1 1, $\mathbf{g}$ tri12, and $\mathbf{h}$ tri14 

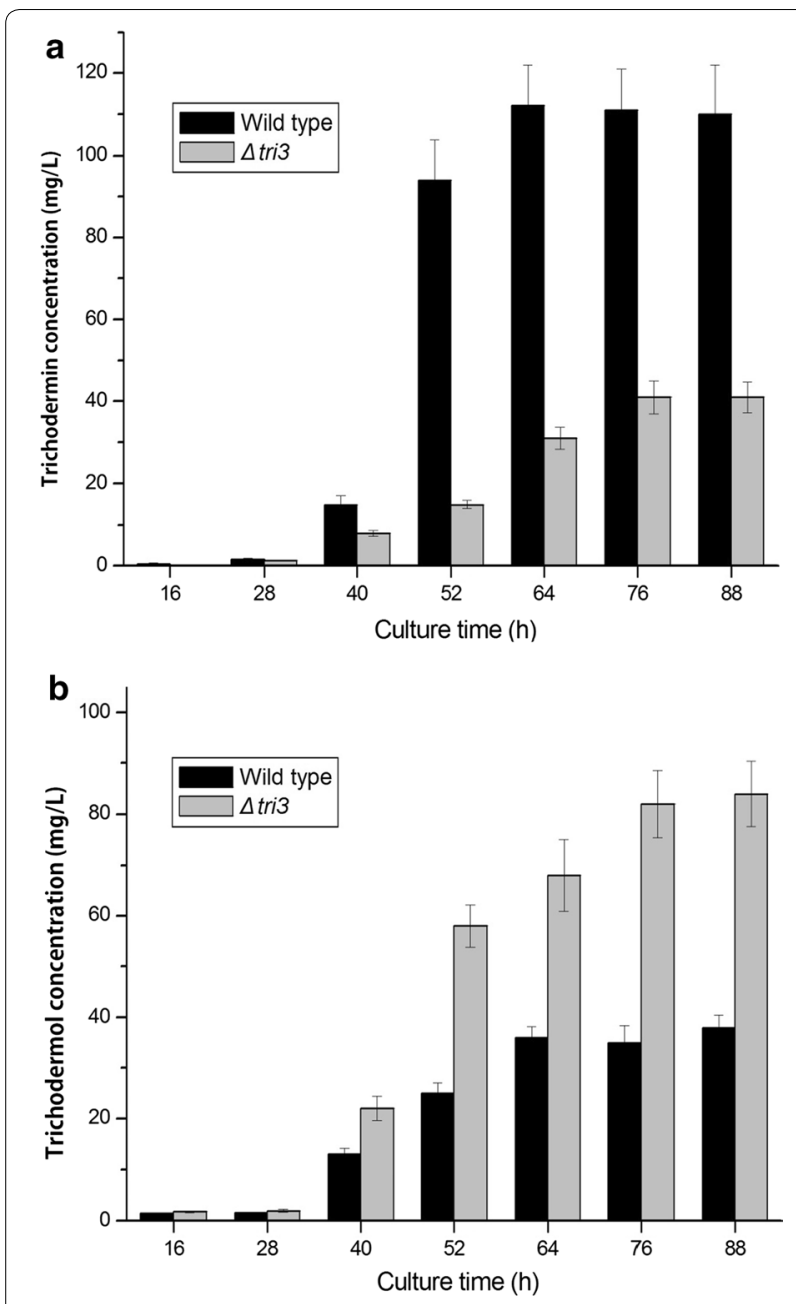

Fig. 6 a Trichodermin production by $\Delta$ tri3 mutants and wild strain 0248. b Trichodermol production by $\Delta$ tri3 mutants and wild strain 0248

Trichoderma species that produces trichodermin, which exhibits strong antifungal activity and has high biotechnological value (Tijerino et al. 2011a, b). Therefore, conducting systematic studies on this species is necessary and urgent.

In this study, tri4 and tri11 deletion mutants were generated to evaluate the roles of tri4 and tri11 in trichodermin biosynthesis. Tatri4 catalyzes the addition of oxygen at $\mathrm{C}-2, \mathrm{C}-12$, and $\mathrm{C}-11$, and this process converts trichodiene to isotrichodiol in T. arundinaceum. Deletion mutant strain $\Delta$ tri4 did not produce trichodermin in strain 0248, and this phenomenon was previously observed for $T$. arundinaceum $\Delta$ tri4 mutants (Cardoza et al. 2011; Malmierca et al. 2012). This result suggested that tri4 is essential for trichodermin biosynthesis in T. brevicompactum. Tatri11 controls the hydroxylation at C-4, and this process converts 12,13-epoxytrichothec-9-ene (EPT) to trichodermol in $T$. arundinaceum. Deletion mutant strain $\Delta$ tri11 also did not biosynthesize trichodermin in T. brevicompactum 0248. Thus, these results first proved the necessity of tri11 for trichodermin biosynthesis in $T$. brevicompactum.

In T. arundinaceum, Tri3 is hypothesized to acetylate oxygen at C-4 of the trichothecene skeleton based on the amino acid sequence and motif, HXXXDG, which is indicative of acetyl-transferases (Murray and Shaw 1997). However, the function of tri3 in Trichoderma has not been verified yet. In our study, $\Delta$ tri3 deletion mutant strain was obtained. The trichodermin in $\Delta$ tri3 cultures was detected using GC, and the results showed lower concentration in the $\Delta$ tri3 deletion mutant than in the wild type. As expected, the tri3 gene was involved in the biosynthesis of trichodermin by $T$. brevicompactum. Furthermore, high abundance of trichodermol in the $\Delta$ tri3 strain was detected, contrary to the quite low trichodermol content in the wild-type culture extracts. These results demonstrated that deletion of tri3 gene in T. brevicompactum 0248 reduced the trichodermin content and accumulated trichodermol. Thus, tri3 gene was responsible for the acetyl moiety to the $\mathrm{C}-4$ oxygen in $T$. brevicompactum.

Small amount of trichodermin was still detected in the $\Delta$ tri3 mutants. This phenomenon was also observed for deletion mutants of Fusarium sporotrichioides (McCormick et al. 1996; Garvey et al. 2009). Trace amounts of trichothecene T-2 toxin were detected in tri3 mutants of $F$ sporotrichioides (McCormick et al. 1996). The small amount of trichodermin suggested the presence of another acetyl-transferase that can convert trichodermol into trichodermin but with lower efficiency. This result implied that an unidentified enzyme analogous to the tri3 acetylase from $T$. brevicompactum strain was probably responsible for the catalysis of the $\mathrm{C}-4$ hydroxyl group of trichodermol. Many unigenes homologous to the acyltransferase-encoding genes were found in our previous transcriptome analysis of T. brevicompactum 0248 (Shentu et al. 2014a). In summary, our results indicated that tri3 gene was responsible for the last step of the acetylation of the C-4 oxygen in T. brevicompactum.

Interestingly, QRT-PCR analyses showed that before $52 \mathrm{~h}$ cultivation, the loss of function of tri3, tri4, or tri11 led to the upregulation of the tri genes. These results were in agreement with the feedback regulation mechanism. To our knowledge, the end-product and intermediate metabolites play a significant role in the regulation of metabolic pathways by directly or indirectly regulating the genes involved in metabolic pathways (Goelzer et al. 2008; Herrgard et al. 2006). Previous studies showed that the synthesis of trichodermin was mainly concentrated during the incubation period of 40-60 h (Yuan et al. 
2016). Therefore, the lower yield of trichodermin in $\Delta$ tri3 mutant or intermediate metabolites in $\Delta$ tri4 and $\Delta$ tri11 mutants may serve as a signal to trigger the feedback regulation. Thus, the expression of tri genes was upregulated, and synthesis of enzymes was induced.

In conclusion, T. brevicompactum is an important species because of its high biocontrol potential. The biosynthesis of trichothecenes by $T$. brevicompactum has been elucidated. It is first proved that tri4 and tri11 are essential for trichodermin biosynthesis by $T$. brevicompactum. This study is also the first to report the function of tri3 in Trichoderma, and the results confirmed the previous hypothesis on the tri3 function in the biosynthesis of trichothecenes.

\section{Additional files}

Additional file 1: Table S1. Primers used to construct plasmid pKT.

Additional file 2: Table S2. Primers used for $q R T-P C R$.

Additional file 3: Table S3. Primers used to verify transformants.

\section{Abbreviations}

PDA: potato-dextrose agar; GC: gas chromatography; ATMT: Agrobacterium tumefaciens-mediated transformation.

\section{Authors' contributions}

$X S, J Y, X F Y, L H$, and FS performed the research, analyzed data, and wrote this manuscript. XPY and $\mathrm{KO}$ designed the research and polished the manuscript. All authors read and approved the final manuscript.

\section{Author details}

${ }^{1}$ Zhejiang Provincial Key Laboratory of Biometrology and Inspection \& Quarantine, College of Life Sciences, China Jiliang University, Hangzhou 310018, Zhejiang, China. ${ }^{2}$ Department of Life Science, Hiroshima Institute of Technology, Hiroshima, Japan.

\section{Acknowledgements \\ Not applicable.}

\section{Competing interests}

The authors declare that they have no competing interests.

\section{Availability of data and materials}

The data supporting the conclusions of this article are included within the article (additional file).

\section{Consent for publication}

Not applicable.

\section{Ethics approval and consent to participate}

Not applicable.

\section{Funding}

The National Key Research and Development Program of China

(2016YFF0202300); National Natural Science Foundation of China (31401793,

31640018); Zhejiang Provincial Programs for Science and Technology Development (2017C32006, 2018C02030); Zhejiang Provincial Natural Science Foundation (LY12C14012).

\section{Publisher's Note}

Springer Nature remains neutral with regard to jurisdictional claims in published maps and institutional affiliations.
Received: 16 October 2017 Accepted: 5 April 2018

Published online: 17 April 2018

\section{References}

Brown DW, Dyer RB, Mccormick SP, Kendra DF, Plattner RD (2004) Functional demarcation of the Fusarium core trichothecene gene cluster. Fungal Genet Biol 41(4):454-462

Cao HP, Wang L, Zhou GX, Wang YY, Chen YL (2014) Rapid assembly of multiple DNA fragments through direct transformation of PCR products into Escherichia coli and Lactobacillus. Plasmid 76:40-46

Cardoza RE, Malmierca MG, Hermosa MR, Alexander NJ, McCormick SP, Proctor $\mathrm{RH}$, Tijerino AM, Rumbero A, Monte E, Gutiérrez S (2011) Identification of loci and functional characterization of trichothecene biosynthesis genes in filamentous fungi of the genus Trichoderma. Appl Environ Microbiol 77:4867-4877

Degenkolb T, Dieckmann R, Nielsen KF, Gräfenhan T, Theis C, Zafari D, Chaverri P, Ismaiel A, Brückner H, Döhren H, Thrane U, Petrini O, Samuels GJ (2008) The Trichoderma brevicompactum clade: a separate lineage with new species, new peptaibiotics, and mycotoxins. Mycol Prog 7:177-219

Dos RMC, Pelegrinelli FMH, Delgado DRT, Furlaneto L, Furlaneto MC (2004) Agrobacterium tumefaciens-mediated genetic transformation of the entomopathogenic fungus Beauveria bassiana. J Microbiol Methods 58:197-202

Garvey GS, Mccormick SP, Alexander NJ, Rayment I (2009) Structural and functional characterization of TR/3 trichothecene 15-O-acetyltransferase from Fusarium sporotrichioides. Protein Sci 18(4):747-761

Goelzer A, Brikci FB, Noirot P, Martin-Verstraete I, Noirot P, Bessières P, Aymerich $\mathrm{S}$, Fromion V (2008) Reconstruction and analysis of the genetic and metabolic regulatory networks of the central metabolism of Bacillus subtilis. BMC Syst Biol 2:20

Harman GE (2006) Overview of mechanisms and uses of Trichoderma spp. Phytopathology 96:190-194

Herrgard MJ, Lee BK, Portnoy VA, Palsson B (2006) Integrated analysis of regulatory and metabolic networks reveals novel regulatory mechanisms in Saccharomyces cerevisiae. Genome Res 16(5):627-635

Kimura M, Tokai T, Takahashi-Ando N, Ohsato S, Fujita M (2007) Molecular and genetic studies of Fusarium trichothecene biosynthesis: pathways, genes, and evolution. Biosci Biotechnol Biochem 71:2105-2123

Kumar A (2010) Overlapping and distinct functions of two Trichoderma virens MAP kinases in cell-wall integrity, antagonistic properties and repression of conidiation. Biochem Biophys Res Commun 398(4):765-770

Lacorte C, Mansur E, Timmerman B, Cordeiro AR (1991) Gene transfer into peanut (Arachis hypogaea L.) by Agrobacterium tumefaciens. Plant Cell Rep 10:354-357

Malmierca MG, Cardoza RE, Alexander NJ, McCormick SP, Hermosa R, Monte E, Gutiérrez S (2012) Involvement of trichoderma trichothecenes in the biocontrol activity and induction of plant defense-related genes. Appl Environ Microbiol 78(14):4856-4868

McCormick SP, Alexander NJ (2007) Myrothecium roridum tri4 encodes a multifunctional oxygenase required for three oxygenation steps. Can J Microbiol 53:572-579

McCormick SP, Hohn TM, Desjardins AE (1996) Isolation and characterization of Tri3, a gene encoding 15-O-acetyltransferase from Fusarium sporotrichioides. Appl Environ Microbiol 62:353-359

Murray IA, Shaw WV (1997) O-acetyltransferases for chloramphenicol and other natural products. Antimicrob Agents Chemother 41:1-6

Nielsen KF, Zafari D, Grafenhan T, Thrane U (2005) Trichothecene production by Trichoderma brevicompactum. J Agric Food Chem 53:8190-8196

Reino JL, Guerrero RF, Hernández-Galán R, Collado IG (2008) Secondary metabolites from species of the biocontrol agent Trichoderma. Phytochem Rev 7:89-123

Shentu XP, Shi YJ, Yu XP (2008) Identification and quantification of trichodermin in fermentation by GC. Chin Pharm J 143:1755-1756

Shentu XP, Liu WP, Zhan XH, Xu YP, Xu JF, Zhang CX (2014a) Transcriptome sequencing and gene expression analysis of Trichoderma brevicompactum under different culture conditions. PLoS ONE 9:e94203 
Shentu XP, Zhan XH, Ma Z, Yu XP, Zhang CX (2014b) Antifungal activity of metabolites of the endophytic fungus Trichoderma brevicompactum from garlic. Braz J Microbiol 45(1):248-254

Szekeres A, Leitgeb B, Kredics L, Antal Z, Hatvani L, Manczinger L, Vagvolgyi C (2005) Peptaibols and related peptaibiotics of Trichoderma. Acta Microbiol Immunol Hung 52:137-168

Tijerino A, Cardoza RE, Moraga J, Malmierca MG, Vicente F, Aleu J, Collado IG, Gutiérrez S, Monte E, Hermosa R (2011a) Overexpression of the trichodiene synthase gene tri5 increases trichodermin production and antimicrobial activity in Trichoderma brevicompactum. Fungal Genet Biol 48:285-296

Tijerino A, Hermosa R, Cardoza RE, Moraga J, Malmierca MG, Aleu J, Collado IG, Monte E, Gutierrez S (2011b) Overexpression of the Trichoderma brevicompactum tri5 gene: effect on the expression of the trichodermin biosynthetic genes and on tomato seedlings. Toxins 3:1220-1232

Wilkins K, Nielsen KF, Din SU (2003) Patterns of volatile metabolites and nonvolatile trichothecenes produced by isolates of Stachybotrys, Fusarium, Trichoderma, Trichothecium and Memnoniella. Environ Sci Pollut Res 10:162-166

Yang L, Yang Q, Sun K, Tian Y, Li H (2011) Agrobacterium tumefaciens-mediated transformation of ChiV gene to Trichoderma harzianum. Appl Biochem Biotechnol 163:937-945

Yuan XF, Shentu XP, Yu XP (2016) Cloning of tri cluster and analysis of tri genes expressions under different Trichodermin-producing conditions in Trichoderma brevicompactum. Chin J Biol Control 32(1):93-100

\section{Submit your manuscript to a SpringerOpen ${ }^{\circ}$ journal and benefit from:}

- Convenient online submission

- Rigorous peer review

- Open access: articles freely available online

- High visibility within the field

- Retaining the copyright to your article

Submit your next manuscript at $\boldsymbol{\nabla}$ springeropen.com 